\title{
Development of Fast Dissolving Oral Films Containing Vitamin B6 for Nausea and Vomiting of Pregnancy (NVP)
}

\author{
K. Jesindha Beyatricks*, Dhananjaya \\ Hillside College of Pharmacy \& Research Centre, Bangalore, India
}

\begin{abstract}
The aim of this study was to formulate and evaluate the oral fast-dissolving film of Vitamin B6 for the effective management of motion sickness and vomiting during pregnancy. Fast-dissolving films were prepared by the solvent-casting method using different polymers, HPMC-15 and Pullulan, along with Propylene glycol as a plasticizer. The Fourier-transform infrared study for the drug-polymer interaction was carried out. Evaluation of physical parameters such as physical appearance, surface texture, uniformity of weight, uniformity of strip thickness, surface pH, folding endurance, uniformity of drug content and percentage of moisture absorption were performed. Kinetic data analysis for the release study and the stability study were also performed. Results of uniformity of weight, thickness, folding endurance, surface pH, percentage drug content, tensile strength and percentage elongation of all the films were found to be satisfactory. The Fourier-transform infrared study indicated that there was no interaction between the drug and the polymers. The in-vitro drug release study showed that a better rate of drug release was achieved by formulations F4 and F8 compared with other formulations. The stability study did not show any significant difference in the external appearance, the drug content and the in-vitro drug release. In conclusion present study suggested that fast dissolving films has a better ability to cross the sublingual barrier at a faster rate, and hence the delivery system was found to be promising as it has the potential of overcoming the drawbacks associated with tablet formulations available in the market presently.
\end{abstract}

Keywords: Fast-dissolving film, Vitamin B6, HPMC-15, Pullulan gum, Mango peel pectin, Crospovidone, solvent casting

Article Info: Received 11 March 2019; $\quad$ Review Completed 20 April 2019; $\quad$ Accepted 23 April 2019; $\quad$ Available online 15 May 2019

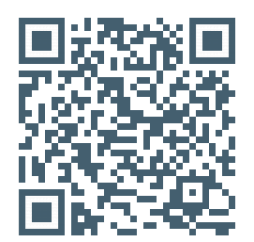

\section{Cite this article as:}

Beyatricks KJ, Dhananjaya, Development of Fast Dissolving Oral Films Containing Vitamin B6 for Nausea and Vomiting of Pregnancy (NVP), Journal of Drug Delivery and Therapeutics. 2019; 9(3):51-59

http://dx.doi.org/10.22270/jddt.v9i3.2735

Dr. K. Jesindha Beyatricks, Professor, Hillside College of Pharmacy \& Research Centre, Bangalore, India

\section{INTRODUCTION}

Oral route is the most preferred route for the delivery of the drugs till date as it bears various advantages over the other route of drug administration, but oral drug delivery systems still need some advancements to be made because of their some drawbacks related to particular class of patients which includes geriatric, pediatric and dysphasic patients associated with many medical conditions as they have difficulty in swallowing or chewing solid dosage forms. Many pediatric and geriatric patients are unwilling to take solid preparations due to fear of choking. ${ }^{1}$ Even with fast dissolving tablets there is a fear of choking due to its tablet type appearance. One study showed that 26\% of 1576 patients experienced difficulty in swallowing tablets. The most common complaint was tablet size, followed by surface form and taste. The problem of swallowing tablets was more evident in geriatric and pediatric patients, as well as travelling patients who may not have ready access to water. ${ }^{2}$

So, fast-dissolving drug-delivery systems came into existence in the late 1970's as an alternative to tablets, capsules and syrups for pediatric and geriatric patients who experience difficulties in swallowing traditional oral solid-dosage forms. These systems consist of the solid dosage forms that disintegrate and dissolve quickly in the oral cavity without the administration of water. Research and development in the oral drug delivery segment has led to transition of dosage forms from simple conventional tablets or capsules to modified release tablets or capsules to oral disintegrating tablet (ODT) to wafer to the recent development of oral fast dissolving films (OFDFs). Amongst the plethora of avenues explored for the rapid drug releasing products, oral strip technology is gaining much attention. ${ }^{3}$

\section{MATERIALS AND METHODS}

Preparation of mouth dissolving oral film by direct solvent casting method

\section{Formulation of fast-dissolving films ${ }^{4}$}

In the present study, fast-dissolving films of Vitamin B6 were prepared by a solvent casting technique. Flat, square-shaped, 
aluminum foil-coated glass molds having a surface area of 25 $\mathrm{cm}^{2}$ were fabricated for casting the films.

\section{Preparation of casting solutions}

The weighed quantities of polymers were kept for swelling overnight in distilled water and dissolved (heated, if necessary). The drug, superdisintegrants and aspartame were dissolved in distilled water and added to the above mentioned polymer solution along with propylene glycol as a plasticizer, mixed thoroughly to form a homogenous mixture. The volume was made up to $10 \mathrm{ml}$ with distilled water. Entrapped air bubbles were removed by applying vacuum.

\section{Preparation of fast-dissolving films ${ }^{5}$}

The casting solution $(10 \mathrm{ml})$ was poured into glass molds and dried at $40^{\circ} \mathrm{C}$ in a vacuum oven for 24 hour for solvent evaporation. The patches were removed by peeling and cut into a square dimension of $5 \mathrm{~cm} \times 5 \mathrm{~cm}\left(25 \mathrm{~cm}^{2}\right)$. These patches were kept in a desiccator for 2 days for further drying and wrapped in aluminium foil, and packed in selfsealing covers. Fast-dissolving films were prepared with different polymers and ratios by maintaining the concentration of the plasticizer and sweetener constant Table 1.

Table 1: Formulation development of Vitamin B6 fast dissolving oral film by solvent casting method

\begin{tabular}{|l|c|c|c|c|c|c|c|c|}
\hline \multicolumn{1}{|c|}{ Formula code } & F1 & F2 & F3 & F4 & F5 & F6 & F7 & F8 \\
\hline Vitamin B6 (mg) & 50 & 50 & 50 & 50 & 50 & 50 & 50 & 50 \\
\hline Crospovidone (mg) & 10 & 20 & -- & -- & 10 & 20 & -- & -- \\
\hline Mango peel pectin (mg) & -- & -- & 10 & 20 & -- & -- & 10 & 20 \\
\hline HPMC-15 (mg) & -- & -- & -- & -- & 60 & 60 & 60 & 60 \\
\hline Pullulan gum (mg) & 60 & 60 & 60 & 60 & -- & -- & -- & -- \\
\hline Propylene glycol (ml) & 0.2 & 0.2 & 0.2 & 0.2 & 0.2 & 0.2 & 0.2 & 0.2 \\
\hline Aspartame (mg) & 20 & 20 & 20 & 20 & 20 & 20 & 20 & 20 \\
\hline Menthol (mg) & Q.S & Q.S & Q.S & Q.S & Q.S & Q.S & Q.S & Q.S \\
\hline Water up to (ml) & 10 & 10 & 10 & 10 & 10 & 10 & 10 & 10 \\
\hline
\end{tabular}

\section{Pre-Formulation Studies}

Preformulation testing is the first step in the rational development of dosage forms of a drug substance. It can be defined as an investigation of physical and chemical properties of a drug substance alone and when combined with excipients. The overall objective of pre-formulation testing is to generate information useful to the formulator in developing stable and bioavailable dosage forms which can be mass produced.

\section{Analytical Method used in the Determination of Vitamin B6}

\section{Identification of pure drug:}

Identification of Vitamin B6 was carried out by Infra-Red Absorption Spectrophotometer.

\section{Determination of $\lambda_{\max }$ :}

From above mentioned standard stock solution, $0.4 \mathrm{ml}$ was withdrawn and added to the $10 \mathrm{ml}$ volumetric flask and diluted up to $10 \mathrm{ml}$ with $0.1 \mathrm{~N} \mathrm{HCl}$. Finally, sample was scanned in the range of $200-400 \mathrm{~nm}$. UV spectrum was recorded and absorption maximum was found to be $292 \mathrm{~nm}$ which is shown in Fig 13 in chapter 5, and was used for the further analytical studies.

\section{Standard calibration curve of Vitamin B6 in 0.1N HCl.9}

From the stock solution $0.2,0.4,0.6,0.8$ and $1.0 \mathrm{ml}$ solution was withdrawn and added in to $10 \mathrm{ml}$ volumetric flask and finally diluted up to $10 \mathrm{ml}$ with $0.1 \mathrm{~N} \mathrm{HCl}$ to get the solution with concentration of $2-10 \mu \mathrm{g} / \mathrm{ml}$ respectively. The absorbance was measured for each solution at $292 \mathrm{~nm}$ using UV-visible spectrophotometer. The graph was plotted for absorbance vs concentration.

\section{Compatibility study:}

Weighed amount of drug (3 mg) was mixed with 100mg of potassium bromide (dried at $40-50^{\circ} \mathrm{C}$ ). The mixture was taken and compressed under 10-ton pressure in a hydraulic press to form a transparent pellet. The pellet was scanned by
IR spectrophotometer. Similar procedure is followed for all relevant excipients used. 10

\section{Evaluation of fast-dissolving films $s^{6,7,8}$}

\section{Mass uniformity and thickness:}

Three randomly selected different films from each batch $\left(2 \times 2 \mathrm{~cm}^{2}\right)$ were weighed individually on an electronic balance. The average weight of the films was calculated. The thickness of the films was measured by vernier caliper at three different positions on the film and the average was calculated.

\section{Drug content uniformity:}

A fast-dissolving film $\left(25 \mathrm{~cm}^{2}\right)$ was transferred into a graduated flask containing $100 \mathrm{ml}$ of distilled water. The flask was shaken for 4 hour in a mechanical shaker. The solution was filtered and after suitable dilutions with distilled water, the absorbance value was measured at 292 $\mathrm{nm}$ using the placebo patch (patch without drug) solution as a blank, and the drug content was calculated.

\section{Folding endurance:}

The folding endurance is expressed as the number of folds (number of times the film is folded at the same place) required to break the specimen or to develop visible cracks. This also gives an indication of brittleness of the film. A strip of $2.5 \mathrm{~cm} \times 2.5 \mathrm{~cm}\left(6.25 \mathrm{~cm}^{2}\right)$ was subjected to folding endurance by folding the patch at the same place repeatedly several times until a visible crack was observed, and the values were reported.

\section{Surface pH:}

The film to be tested was placed in a Petri dish and was moistened with $0.5 \mathrm{ml}$ of distilled water and kept for $30 \mathrm{sec}$. The $\mathrm{pH}$ was noted after bringing the electrode of the $\mathrm{pH}$ meter in contact with the surface of the formulation and allowing equilibration for $1 \mathrm{~min}$. The average of three determinations for each formulation was done. 


\section{Elongation and tensile strength:}

This mechanical property was evaluated using the Instron universal testing instrument with a $5 \mathrm{~kg}$ load cell. Film strips in a special dimension and free from air bubbles or physical imperfections were held between two clamps positioned at a distance of $3 \mathrm{~cm}$. During measurement, the strips were pulled by the top clamps at a rate of $100 \mathrm{~mm} / \mathrm{min}$; the force and elongation were measured when the film broke. Results from film samples, which broke at and not between the clamps, were not included in the calculations. Measurements were run in triplicate for each film. Two mechanical properties, namely, tensile strength and percentage elongation were computed for the evaluation of the film. Tensile strength is the maximum stress applied to a point at which the film specimen breaks and can be computed from the applied load at rupture.

\section{Disintegration test:}

Disintegration test was performed to ensure the disintegration of the film in water. One film from each formulation was introduced into one tube of disintegration apparatus IP. A disc was added into the tube. The assembly was suspended in a beaker containing simulated saliva and the apparatus was operated until the film disintegrated.

\section{In-vitro dissolution studies: 11}

The simulated salivary fluid was taken as the dissolution medium to determine the drug release.

The dissolution profile of quick release films of Vitamin B6 was carried out in a beaker containing $30 \mathrm{ml}$ of the simulated salivary fluid ( $\mathrm{pH} \mathrm{6.8)}$ as a dissolution medium, maintained at $37 \pm 0.5^{\circ} \mathrm{C}$. The medium was stirred at $100 \mathrm{rpm}$. Aliquots $(5 \mathrm{ml})$ of the dissolution medium were withdrawn at 2, 4, 8, 12 and 15 minute time intervals and the same amount was replaced with the fresh medium. Samples were assayed spectrophotometrically at $292 \mathrm{~nm}$. Three trials were carried out for all the samples and the average value was taken. The percentage of the drug dissolved at various time intervals was calculated and plotted against time.

\section{Mathematical modelling of drug release profile: 12}

The cumulative amount of Vitamin B6 release from the formulated tablets at different time intervals were fitted to zero order kinetics, first order kinetics, Higuchi's model and korsmeyer-peppas model to characterize mechanism of drug release.

\section{Zero Order Kinetics}

It describes the system in which the release rate is independent of its concentration.

$$
\mathbf{Q}=\mathbf{Q}_{\mathbf{0}}+\mathbf{K}_{\mathbf{0}} \mathbf{t}
$$

Where, $\mathbf{Q t}=$ amount of drug dissolved in time $t, \mathbf{Q}_{\mathbf{0}}=$ initial amount of drug in the solution

$$
\mathbf{K}_{\mathbf{0}}=\text { zero order release constant }
$$

If the zero order drug release kinetic is obeyed, a plot of $Q_{t}$ versus $t$ will give straight line with a slope of $K_{0}$ and an intercept at $\mathrm{Q}_{0}$.

\section{First Order Kinetic:}

It describes the drug release from the system in which the release rate is concentration dependant. ${ }^{56}$

$$
\log Q_{t}=\log Q_{0}+K_{1} t / 2.303
$$

Where, $\mathrm{Qt}=$ amount of drug dissolved in time
$\mathrm{K}_{1}=$ first order release constant

If the release pattern of drug follows first order kinetics, then a plot of $\log \left(Q_{0}-Q_{t}\right)$ versus $t$ will be straight line with a slope of $\mathrm{K}_{1} / 2.303$ and an intercept at $\mathrm{t}=0$ of $\log \mathrm{Q}_{0}$.

\section{Higuchi Model}

It describes the fraction of drug release from a matrix is proportional to square root of time.

\section{$M t / M \infty=K_{H} t^{1 / 2}$}

Where, Mt and M $\infty$ are cumulative amount of drug release at time $t$ and infinite time, and

$\mathrm{K}_{\mathrm{H}}=$ Higuchi dissolution constant reflection formulation characteristics.

If the Higuchi model of drug release is obeyed, then a plot of $\mathrm{Mt} / \mathrm{M} \infty$ versus $\mathrm{t}^{1 / 2}$ will be straight line with slope of $\mathrm{K}_{\mathrm{H}}$.

\section{Korsmeyer- Peppas Model}

The power law describes the fractional drug release is exponentially related to the release time and adequately describes the release of drug from slabs, cylinders and spheres, as expressed in following equation.

$$
\text { Mt } / \mathbf{M} \infty=\mathrm{K} \mathrm{t}^{\mathbf{n}}
$$

$$
\log (M t / M \infty)=\log K+n \log t
$$

\section{Stability Studies ${ }^{13}$}

Stability can be defined as the capacity of drug product to remain within specifications established to ensure its identity, strength, quality, and purity.

\section{Importance of stability studies}

Stability studies are important for the following reasons.

1. This is an assurance given by the manufacturer that the patient would receive a uniform dose throughout the shelf life.

2. The drug control administration insists on manufacturers on conducting the stability studies, identity, strength, purity and quality of the drug for an extended period of time in the conditions of normal storage.

3. Stability testing prevents the possibility of marketing an unstable product. Both physical and chemical degradation of drug can result in unstable product.

The optimized formulation was subjected for two months stability study according to ICH guidelines. The selected formulations were packed in aluminium foil in tightly closed container. They were then stored at $40^{\circ} \mathrm{C} / 75 \% \mathrm{RH}$ for two months and evaluated for their permeation study.

\section{RESULTS AND DISCUSSION}

\section{Standard graph of Vitamin B6}

$\lambda_{\max }$ of Vitamin B6 was found to be $292 \mathrm{~nm}$ as it shows maximum absorbance in this wavelength.

\subsection{Calibration curve of Vitamin B6}

The absorbance was measured in a UV spectrophotometer at $292 \mathrm{~nm}$ against $0.1 \mathrm{~N} \mathrm{HCl}$. The absorbance so obtained was plotted and shown in the Figure 1. 


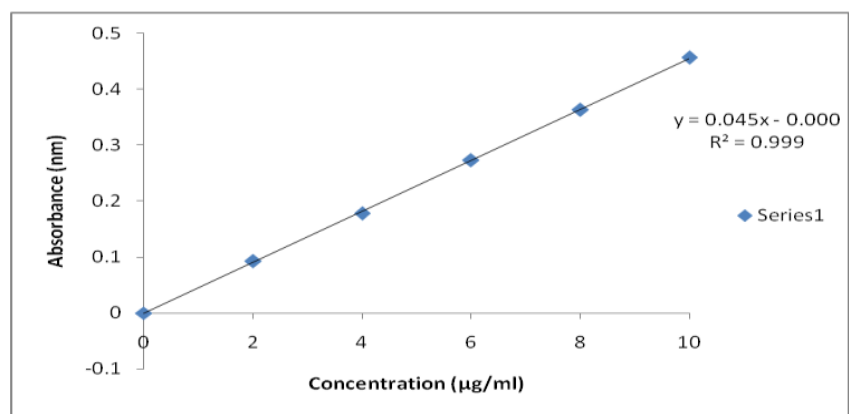

Figure 1: Calibration Curve of Vitamin B6 in $0.1 \mathrm{~N} \mathrm{HCl}$

\section{Compatibility studies using FTIR}

Infra-red spectrum of drug and polymers were recorded over $\mathrm{KBr}$ disc method and obtained spectra were shown in the Figure 2-6.

All the characteristic peaks of Vitamin B6 were present in the spectrum of drug and polymer mixture, indicating compatibility between drug and polymer. The spectrum confirmed that there is no significant change in the chemical integrity of the drug. There is no change in functional group peaks $(\mathrm{C}=\mathrm{O}, \mathrm{C}=\mathrm{N}, \mathrm{C}-\mathrm{S}, \mathrm{C}-\mathrm{N}, \mathrm{S}=\mathrm{O}, \mathrm{C}-\mathrm{Cl})$ of Vitamin $\mathrm{B} 6$ in all the IR-spectra.

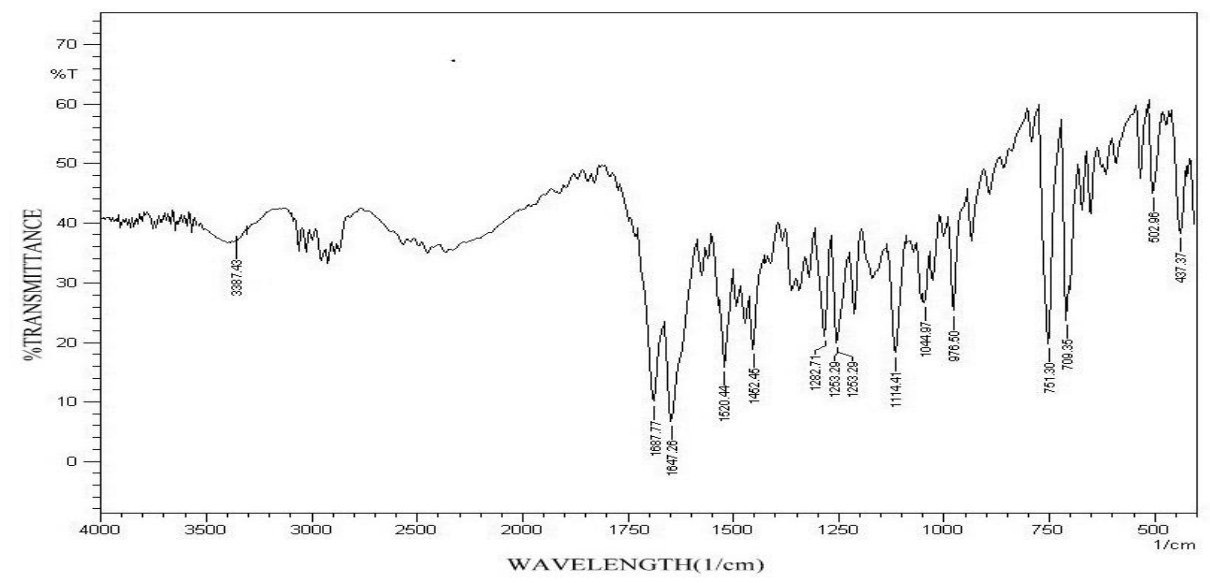

Figure 2: FT-IR spectrum of pure drug (Vitamin B6)

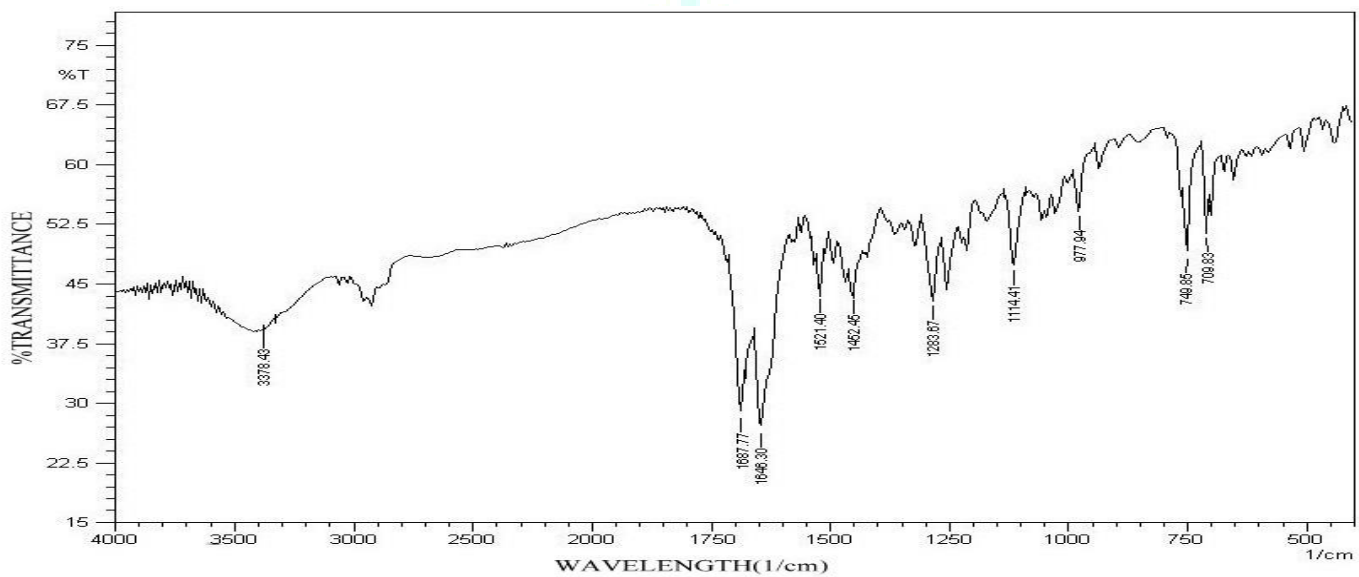

Figure 3: FT-IR of formulation F1

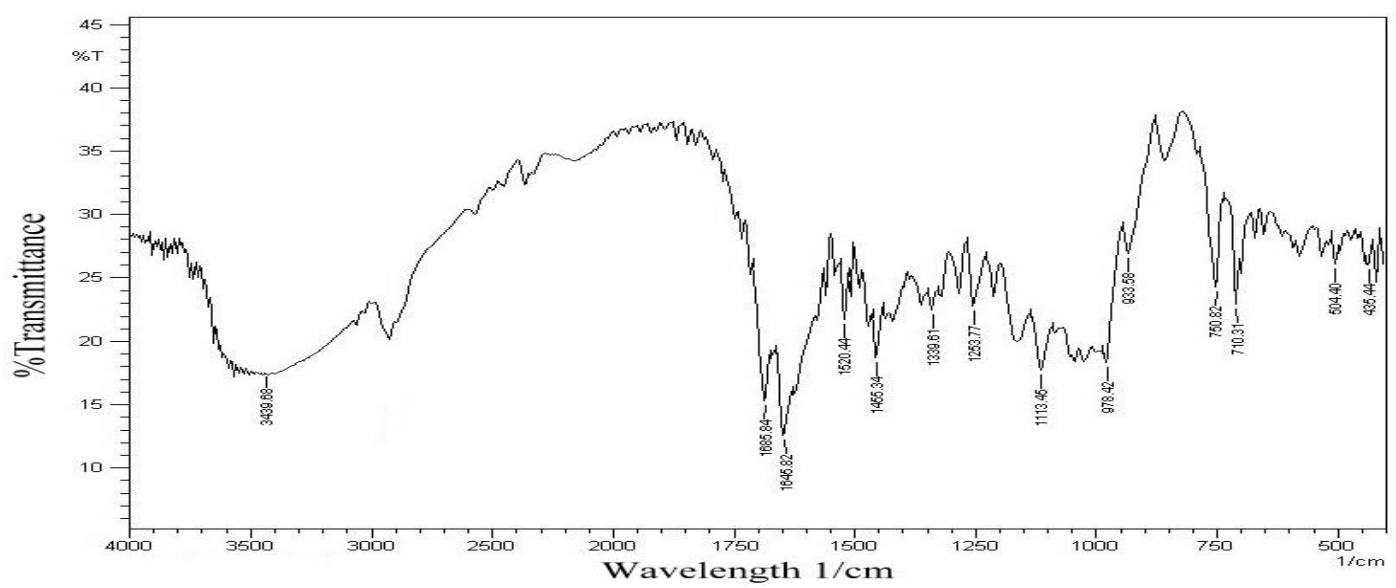

Figure 4: FT-IR spectrum of formulation F3 


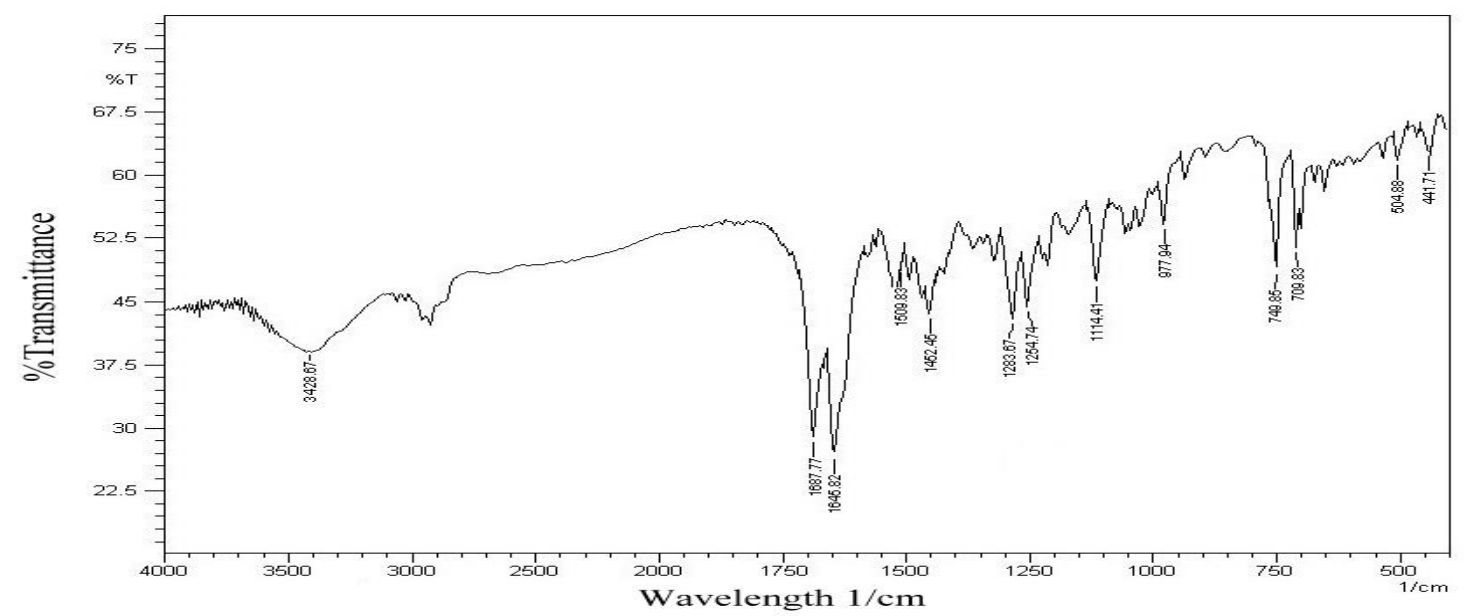

Figure 5: FT-IR spectrum of formulation F5

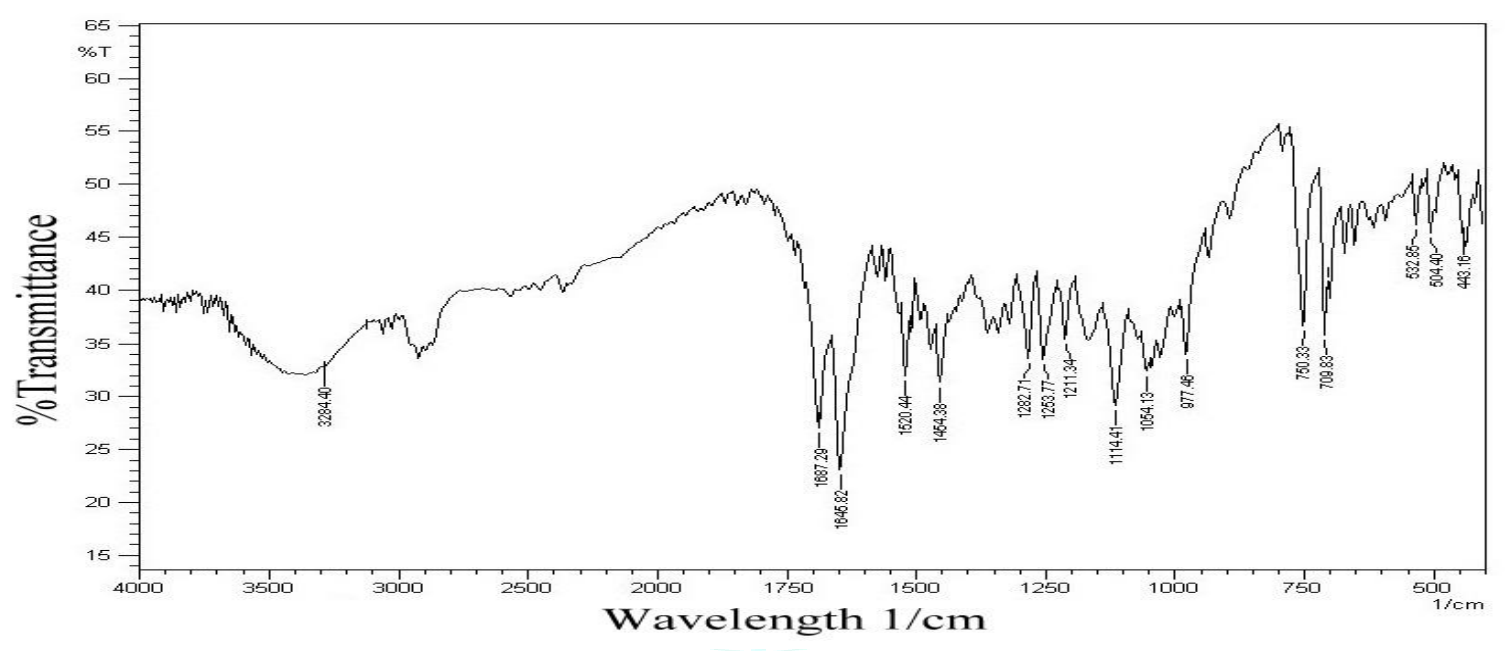

Figure 6: FT-IR Spectrum of formulation F7

\section{Preparation of film formulations:}

Fast-dissolving films of Vitamin B6 were prepared by the solvent casting method on glass molds, using Pullulan gum and HPMC-15 as polymers. Propylene glycol was used as a plasticizer and aspartame as a sweetener. Distilled water was used as a solvent for HPMC and Pullulan gum. Mango peel pectin and crospovidone was used as superdisintegrants. The effect of the concentration ratio of polymers and superdisintegrants was studied by preparing various formulations of fast-dissolving films. In the preparation, the addition of ingredients, particularly propylene glycol and aspartame, was followed after the careful evaluation of films for physical characteristics. In all these formulations, a constant amount of drug (50 mg) was maintained. The casting solution $(10 \mathrm{ml})$ was poured into 25 $\mathrm{cm}^{2}$ molds, so that each square centimetre contains approximately $2 \mathrm{mg}$ of the drug. The concentration of polymers $\mathrm{s}$ and the concentration of other ingredients such as plasticizer and sweetener were kept constant. A total number of eight formulations were prepared.

\section{Evaluation of Vitamin B6 fast dissolving oral film:}

\section{Mass uniformity, thickness and appearance:}

From the results of the tests for physical characterization conducted, it is observed that the weight and thickness of all film samples was uniform within each formulation. The thickness of 12 films of each formulation was determined using a micrometer screw gauge, and the average thickness was determined. The values were found to be in the range of $120-210 \mu \mathrm{m}$, which is said to be acceptable for fastdissolving films. The films with HPMC as a polymer showed a slightly increase in thickness which might be due to increased viscosity of HPMC. Physical appearance of prepared film was checked simply with visual infection of films and by feel or touch. Films formulated from Pullulan gum were smooth, flexible and transparent whereas those prepared from HPMC-15 were slightly rough in texture, less flexible and translucent. The results were showed in Table 3.

\section{Drug content uniformity:}

The drug content estimation was done using by UV spectrophotometer (Shimadzu UV-1800), at 292nm. Drug content of all the formulations were found between 96.81 to $99.07 \%$. All the film formulations of Vitamin B6 showed uniform drug content as shown in Table 3.

\section{Folding endurance:}

The result of folding endurance is shown in Table 3. Folding endurance of fast dissolving film was found in the range $250 \pm 3$ to $454 \pm 3$. Fast dissolving film containing Pullulan gum as polymer showed optimum folding endurance (more than $400 \pm 2$ ) compared to formulations containing HPMC-12. Therefore on the basis of appearance and folding endurance, we can say that Pullulan gum based formulations were superior to HPMC-12 based formulations. 
Table 3: Evaluation of mouth dissolving film of Vitamin B6

\begin{tabular}{|c|c|c|c|c|c|}
\hline Formulation & $\begin{array}{c}\text { Mass uniformity } \\
(\mathbf{m g})\end{array}$ & $\begin{array}{c}\text { Thickness } \\
(\boldsymbol{\mu m})\end{array}$ & Appearance & Drug content & $\begin{array}{c}\text { Folding } \\
\text { endurance }\end{array}$ \\
\hline F1 & 89 & $110 \pm 3.5$ & Transparent & $98.81 \pm 0.002$ & $>400$ \\
\hline F2 & 94 & $135 \pm 05$ & Transparent & $98.71 \pm 0.022$ & $>400$ \\
\hline F3 & 87 & $210 \pm 05$ & Transparent & $97.76 \pm 0.021$ & $>400$ \\
\hline F4 & 98 & $195 \pm 4.5$ & Transparent & $99.07 \pm 0.015$ & $>400$ \\
\hline F6 & 89 & $160 \pm 05$ & Translucent & $98.81 \pm 0.003$ & $<300$ \\
\hline F7 & 88 & $165 \pm 03$ & Translucent & $96.81 \pm 0.032$ & $<300$ \\
\hline F8 & 95 & $184 \pm 2.5$ & Translucent & $98.46 \pm 0.042$ & $<300$ \\
\hline
\end{tabular}

\section{Surface pH:}

An acidic or alkaline $\mathrm{pH}$ of administered dosage forms can irritate the buccal mucosa. The surface $\mathrm{pH}$ of the prepared fist dissolving film was found in the range of $6.7 \pm 0.3$ to $7.02 \pm 03$. The measured surface $\mathrm{pH}$ was found to be close to neutral in all the formulations which means that they have less potential to irritate the buccal mucosa and therefore they should be fairly comfortable and hence, more acceptable by the patients.

\section{Elongation and tensile strength:}

The tensile strength gives an indication of the strength and the elasticity of the film reflected by the parameters tensile strength and elongation at break. A weak and soft polymer is characterized by low tensile strength and elongation at break, a hard and brittle polymer shows a moderate tensile strength and low elongation at break and a soft and tough polymer shows a high tensile strength and elongation at break. In this study, the effect of superdisintegrants to polymer ratio was observed. The percentage elongation increased with the decrease in the percentage of superdisintegrant to polymer ratio. Among the formulation batches, HPMC based films showed a better tensile strength due to the hydrophobic nature of the polymers compared to Pullulan gum. The percentage elongation of prepared film was found in the range of $36.88 \pm 1.3 \%$ to $72.15 \pm 1.4 \%$ and Tensile strength was found in the range of $1.28 \pm 0.06$ $\mathrm{kg} / \mathrm{mm}^{2}$ to $1.41 \pm 0.017 \mathrm{~kg} / \mathrm{mm}^{2}$. Results of tensile strength and percentage elongation are shown in Table 4.

Table 4: Evaluation of mouth dissolving film of Vitamin B6

\begin{tabular}{|c|c|c|c|c|}
\hline Formulation & Surface pH & $\begin{array}{c}\text { Tensile strength } \\
\mathbf{K g} / \mathbf{m m}^{\mathbf{2}}\end{array}$ & $\begin{array}{c}\mathbf{\%} \\
\text { Elongation }\end{array}$ & $\begin{array}{c}\text { Disintegration } \\
\text { time (sec) }\end{array}$ \\
\hline F1 & $6.70 \pm 0.3$ & $1.28 \pm 0.006$ & $36.88 \pm 1.30$ & 72 \\
\hline F2 & $6.92 \pm 0.3$ & $1.31 \pm 0.016$ & $43.75 \pm 1.74$ & 44 \\
\hline F3 & $6.80 \pm 0.3$ & $1.29 \pm 0.022$ & $55.86 \pm 1.55$ & 49 \\
\hline F4 & $7.02 \pm 0.3$ & $1.32 \pm 0.054$ & $47.11 \pm 1.63$ & 33 \\
\hline F5 & $6.72 \pm 0.3$ & $1.40 \pm 0.015$ & $72.15 \pm 1.40$ & 51 \\
\hline F6 & $6.94 \pm 0.3$ & $1.36 \pm 0.047$ & $65.88 \pm 1.34$ & 47 \\
\hline F7 & $7.01 \pm 0.3$ & $1.37 \pm 0.018$ & $69.05 \pm 1.52$ & 54 \\
\hline F8 & $6.85 \pm 0.3$ & $1.39 \pm 0.024$ & $58.19 \pm 1.54$ & 38 \\
\hline
\end{tabular}

\section{Disintegration test:}

The disintegration time of prepared fast dissolving film was ranging between 33 second to 72 second and results shown in Table 4. Disintegration time of the films was decreased when the concentration of superdisintegrants were increased. Among the superdisintegrants used, formulation F4 containing 1:3 ratio of Mango peel pectin and Pullulan gum showed rapid disintegration i.e. 33 second. Fast disintegration time of formulation F4 might be due to higher swelling index of Mango peel pectin.

\section{In-vitro dissolution studies:}

From the in-vitro drug release, it was observed that formulations F4 containing Pullulan gum in combination with higher concentration of Mango peel pectin as a superdisintegrant showed faster drug release, and among the HPMC based films, formulation batch F8 showed more than $90 \%$ of drug release with in 15 minute. The release of the drug from prepared film was dependent on concentration of superdisintegrants used, when the concentration of superdisintegrants is increased, drug release from formulation was also increased, and this might be due to faster disintegration time for these formulations. The drug release was found to be in the following order: F4 > F8 $>$ F6 $>$ F2 $>$ F $3>$ F7 $>$ F5 $>$ F1. The release profile of fastdissolving films was compared with that of conventional marketed product and it was observed that the drug release from fast-dissolving films was much faster than that from formulation. Results of in-vitro drug release studies were showed in Table 5 and Figure 7. 
Table 5: In-vitro drug release studies of Vitamin B6 fast dissolving films

\begin{tabular}{|c|c|c|c|c|c|c|c|c|c|}
\hline \multirow{3}{*}{$\begin{array}{c}\text { Time } \\
\text { in } \\
\text { mins }\end{array}$} & \multicolumn{8}{|c|}{$\%$ cumulative drug release } & \multirow[b]{3}{*}{$\begin{array}{c}\text { Marketed } \\
\text { product }\end{array}$} \\
\hline & \multicolumn{8}{|c|}{ Formulation code } & \\
\hline & F1 & F2 & F3 & F4 & F5 & F6 & F7 & F8 & \\
\hline 0 & 0 & 0 & 0 & 0 & 0 & 0 & 0 & 0 & 0 \\
\hline 2 & 14.26 & 17.04 & 15.56 & 33.34 & 16.02 & 18.63 & 16.56 & 28.45 & 8.32 \\
\hline 4 & 29.08 & 39.17 & 34.08 & 57.94 & 26.95 & 42.05 & 30.19 & 54.19 & 19.44 \\
\hline 8 & 41.79 & 47.12 & 46.94 & 74.59 & 43.79 & 58.89 & 44.28 & 67.01 & 28.57 \\
\hline 12 & 54.67 & 68.14 & 66.57 & 85.26 & 55.16 & 70.64 & 57.16 & 74.46 & 41.84 \\
\hline 15 & 65.91 & 83.59 & 80.17 & 98.81 & 72.37 & 92.38 & 76.10 & 95.10 & 59.88 \\
\hline
\end{tabular}

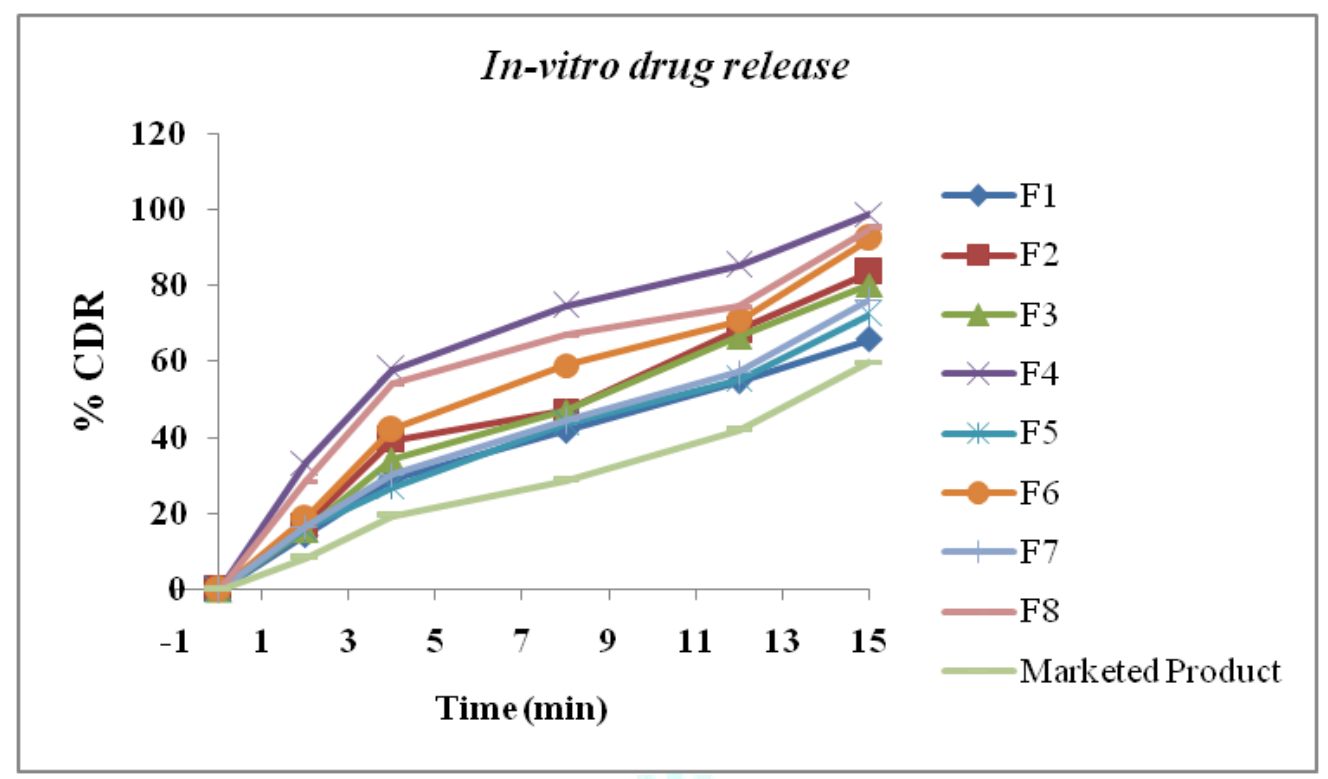

Figure 7: In-vitro drug release studies of Vitamin B6 fast dissolving films

\section{Drug Release Kinetics:}

Investigation of the drug release from the fast dissolving film was done by plotting the data of in-vitro release studies into different kinetic equations (Zero order, First order, and Higuchi's equation). The release mechanism was understood by fitting the data to Korsemeyer Peppas model. The results of different release kinetics pattern and release mechanism of the drug from the different fast dissolving film formulations were showed in Table 6.

From the kinetic studies, Table 6 the examination of correlation coefficient ' $r$ ' indicated that the drug release followed first order kinetics. It was found that the value of ' $r$ ' for first order ranged from $0.989-0.998$, which is near to 1 when compare to zero order (0.761-0.893) and Higuchi square root (0.598-0.796). So, it was understood to be following first order release pattern by the prepared film. In the present investigation, release from the hydrophilic polymers followed the combination of diffusion and erosion as the ' $n$ ' values ranged from 0.465 to 0.625 as per the Korsmeyer and Peppas model, which in turn justified the suitability of polymers for the preparation of fast-dissolving films. A result of release kinetics studies is showed in Table 6.

Table 6: Release exponent values and release rate constant values for different fast dissolving film formulations

\begin{tabular}{|c|c|c|c|c|c|}
\hline \multirow{2}{*}{ Formula Code } & \multicolumn{2}{|l|}{ Korsmeyar and Peppas } & Higuchi & First Order & Zero Order \\
\cline { 2 - 6 } & $\mathbf{R}^{\mathbf{2}}$ & $\mathbf{N}$ & $\mathbf{R}^{\mathbf{2}}$ & $\mathbf{R}^{\mathbf{2}}$ & $\mathbf{R}^{\mathbf{2}}$ \\
\hline F1 & 0.850 & 0.465 & 0.674 & 0.987 & 0.828 \\
\hline F2 & 0.842 & 0.559 & 0.598 & 0.990 & 0.892 \\
\hline F3 & 0.846 & 0.547 & 0.688 & 0.998 & 0.844 \\
\hline F4 & 0.825 & 0.625 & 0.686 & 0.984 & 0.761 \\
\hline F6 & 0.823 & 0.535 & 0.795 & 0.992 & 0.807 \\
\hline F7 & 0.835 & 0.486 & 0.693 & 0.984 & 0.893 \\
\hline F8 & 0.840 & 0.496 & 0.792 & 0.985 & 0.838 \\
\hline
\end{tabular}

$\mathbf{R}^{2}=$ Regression coefficient, $\mathbf{n}=$ Exponential value 


\section{Stability studies:}

Based on the results of in-vitro release studies, formulation F4 and F8 were selected for short term stability study. Stability studies were carried out for 90 days at $45-50^{\circ} \mathrm{C}$ $(75 \% \mathrm{RH})$ and $25-30^{\circ} \mathrm{C}(60 \% \mathrm{RH})$. The films were observed for physical changes, the percentage drug content and the percentage drug release. Fast-dissolving films of Vitamin B6 were found to be physically and chemically stable and showed no significant change in terms of physical characteristics, the percentage drug content and the percentage drug release.

Table 7: Results of short term stability of formulation F4 and F5 at $40^{\circ}-50^{\circ} \mathrm{C} / 75 \% \mathrm{RH}$

\begin{tabular}{|c|c|c|c|c|}
\hline \multirow{2}{*}{$\begin{array}{c}\text { Times in } \\
\text { (Days) }\end{array}$} & Drug content & \% CDR & Drug content & \% CDR \\
\cline { 2 - 5 } Initial & 99.07 & 98.81 & 98.68 & 95.10 \\
\hline 15 & 99.04 & 98.80 & 98.61 & 95.00 \\
\hline 30 & 99.01 & 98.74 & 98.54 & 94.98 \\
\hline 45 & 98.89 & 98.70 & 98.47 & 94.95 \\
\hline 60 & 98.86 & 98.52 & 98.44 & 94.87 \\
\hline 90 & 98.82 & 98.21 & 98.42 & 94.84 \\
\hline
\end{tabular}

Table 8: Results of short term stability of formulation F4 and F5 at $25^{\circ}-30^{\circ} \mathrm{C} / 60 \% \mathrm{RH}$

\begin{tabular}{|c|c|c|c|c|}
\hline \multirow{2}{*}{$\begin{array}{c}\text { Times in } \\
\text { (Days) }\end{array}$} & \multicolumn{2}{|c|}{ F4 Formulation } & \multicolumn{2}{c|}{ F8 Formulation } \\
\cline { 2 - 5 } & Drug content & \% CDR & Drug content & \% CDR \\
\hline Initial & 99.07 & 98.81 & 98.68 & 95.10 \\
\hline 15 & 99.07 & 98.81 & 98.67 & 95.10 \\
\hline 30 & 99.05 & 98.78 & 98.63 & 94.99 \\
\hline 45 & 99.04 & 98.74 & 98.60 & 94.96 \\
\hline 60 & 98.98 & 98.70 & 98.56 & 94.93 \\
\hline 90 & 98.93 & 98.65 & 98.52 & 94.90 \\
\hline
\end{tabular}

\section{CONCLUSION}

Oral drug delivery is currently the golden standard in the pharmaceutical industry where it is regarded as the safest, most convenient and an economical method of drug delivery having the highest patient compliance.

The most popular dosage forms being tablets and capsules, one important drawback of these dosage forms however is the difficulty to swallow. Dysphagia or difficult in swallowing is seen to affect nearly $35 \%$ of the population. This disorder is also associated with number of medical conditions. Many elder persons will have difficulty in taking conventional dosage forms. However, geriatric, pediatric and mentally ill patients experience difficulty in swallowing conventional tablets, which leads to poor patient compliance. In some cases such as motion sickness, sudden episode of allergic attack or coughing and unavailability of water for swallowing tablets may become difficult.

To overcome this hurdles, scientists have developed innovative drug delivery systems known as fast dissolving dosage form. United States Food and drug administration (FDA) defined fast dissolving oral film is defined as "an ultrathin film containing active ingredient that dissolves or disintegrates in the saliva at a remarkably fast rate, within few seconds without the aid of water or chewing". The faster the drug is going in to solution, the quicker the absorption and onset of clinical effect. Some drugs, which are soluble in saliva rapidly, absorbed from the mouth, pharynx and Oesophagus as the saliva passes down into the stomach thus avoids first pass metabolism and enhances the bioavailability.

For the present study, we have selected Vitamin B6 supplements have been used for years by conventional physicians as a treatment for morning sickness.
The main aim of this work is to prepare and evaluate of fast dissolving film of Vitamin B6 using superdisintegrants like Crospovidone and Mango peel pectin and HPMC-15 and Pullulan gum as polymer. Eight formulations of fast dissolving film of Vitamin B6 were prepared by solvent casting method and subjected to various evaluation tests.

All the fast dissolving films were evaluated for compatibility, thickness, folding endurance, tensile strength, disintegration time and in-vitro drug release studies. Further the fast dissolving film formulations were subjected to their short term stability studies for three months and again subjected to further evaluation tests.

Results revealed that all the formulated films showed acceptable thickness, percentage elongation, and physical properties. In-vitro release studies revealed that, the drug released by $\mathrm{F} 4$ formulation was comparatively higher release than the other formulations. The formulation F4 shows $98.81 \%$ of drug release at the end of $15 \mathrm{~min}$. To analyze the drug release mechanism from the fast dissolving film, the invitro drug release data was fitted to Zero order, First order, Higuchi's model equation and Korsmeyer-Peppas model. It was observed that the release of drug followed First order release kinetics and ' $n$ ' value indicates that release mechanism follows non-Fickian.

Thus, it can be summarized that stable Vitamin B6 fast dissolving film were prepared successfully by using superdisintegrants in different ratio by solvent casting technique. The prepared fast dissolving films disintegrates in matter of seconds without need of water and enhances the absorption, this leads to increase in the bioavailability of method to enhance the dissolution rate. 


\section{REFERENCES}

1. Habib W, Pritchard JF, Bozigian HP, Gooding AE, Griffin RH, Mitchell R. Fast-dissolve drug delivery system. Crit Rev Ther Drug Carrier Syst. 2000; 17: 61-72.

2. Liang CA, Chen HL, Fast dissolving intraoral drug delivery systems. Expert Opin Ther Pate. 2001;11: 981-6.

3. Goel H, Rai P, Rana V, Tiwary KA. Orally disintegration systems: Innovations in formulation and technology. Recent Pate Drug Deliv Form. 2008; 2:258-74.

4. Ali MS, Vijendar C, Kumar SD, Krishnaveni J. Formulation and evaluation of fast dissolving oral films of diazepam. J Pharmacovigilence. 2016; 4(3):1-5.

5. Koradia DK, Sidhdhapara TP, Sheth RN. Preparation and optimization of fast dissolving film of naratriptan hydrochloride. Recent Patents Drug Del Form. 2017; 11(2):124-31.

6. Narwal S, Saini V. Formulation, development and evaluation of fast disintegrating thin film of esomeprazole magnesium trihydrate. Am J Pharm Tech Res. 2016; 6(4):519-40.

7. Shah C, Rathod K, Sanghvi K. Formulation development and optimization of rapid dissolving film of Olanzapine. Int J Pharm Res Bio-Sci. 2014; 3(2):555-69.
8. Saini S, Nanda A, Dhari J. Formulation, development and evaluation of oral fast dissolving anti-allergic film of levocetrizine dihydrochloride. Pharm Sci Res. 2011; 3(7):13225.

9. Sharma YR. Elementary organic spectroscopy, principles and chemical application. 1st ed. S. Chand publication; 2001:p.8182.

10. Ahmed MG, Narayana CR, Harish NM, Prabhu P, Roopesh PT. Polymeric strips containing sparfloxacin for the long term treatment of periodontitis. Int J Pharm Res. 2008:48-53

11. Kanakadurga ND, Rani AP, Mrudula SB. Formulation and evaluation of oral disintegrating tablets of Montelukast sodium: effect of functionality of superdisintegrants. J Pharma Res. 2010; 3(4):803-8.

12. Bhaskaran S. Industrial pharmacy. $1^{\text {st }}$ ed. New Delhi. Birla Publications, Pvt. Ltd; 2010. p. 46-69.

13. ICH Q1A (R2) Stability testing guidelines: Stability testing of new drug substances and products. [Online]. [Cited 20017 nov 10]; available form: URL:

http://www.tga.health.gov.au/docs/pdf/euguide/inch/27369 9r2en.pdf 